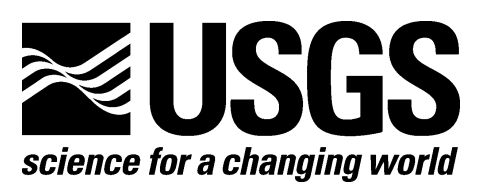

\title{
Development and Evaluation of a Riparian Buffer Mapping Tool
}

By Lesley E. Milheim and Peter R. Claggett

Open-File Report Series 2008-1266

U.S. Department of the Interior

U.S. Geological Survey 


\section{U.S. Department of the Interior DIRK KEMPTHORNE, Secretary}

\section{U.S. Geological Survey \\ Mark D. Myers, Director}

\section{U.S. Geological Survey, Reston, Virginia: 2008}

For product and ordering information:

World Wide Web: http://www.usgs.gov/pubprod

Telephone: 1-888-ASK-USGS

For more information on the USGS - the Federal source for science about the Earth, its natural and living resources, natural hazards, and the environment:

World Wide Web: http://www.usgs.gov

Telephone: 1-888-ASK-USGS

Suggested citation:

Milheim, L.E., and Claggett, P.R., 2008, Development and evaluation of a riparian buffer mapping tool: U.S. Geological Survey Open-File Report 2008-1266, 15 p., available only online at

http://pubs.usgs.gov/of/2008/1266/.

Any use of trade, product, or firm names is for descriptive purposes only and does not imply endorsement by the U.S. Government.

Although this report is in the public domain, permission must be secured from the individual copyright owners to reproduce any copyrighted material contained within this report. 


\section{Contents}

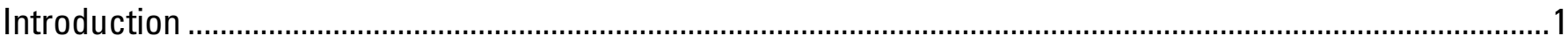

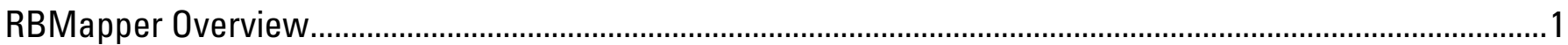

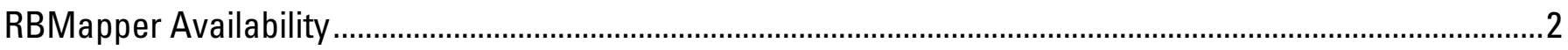

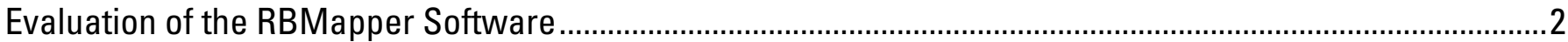

Strengths

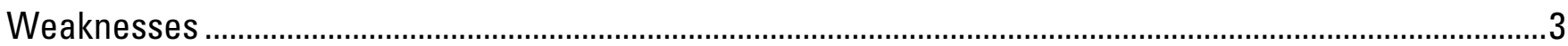

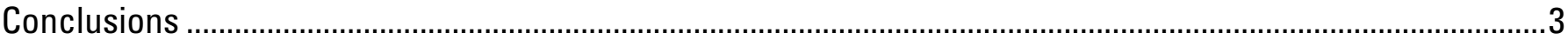

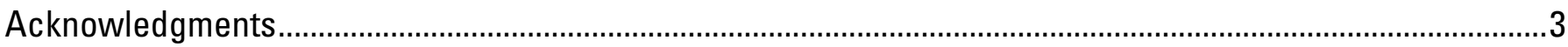

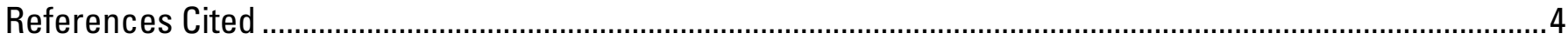

Appendix 1—RBMapper Evaluation and Reviewer Comments ........................................................................

\section{Figures}

1. RBMapper interface displaying the tutorial image with the windows for setting preferences open. Tutorial image courtesy of GeoEye

2. RBMapper interface displaying the results of the water detection function using default settings. Image courtesy of GeoEye.

3. RBMapper interface displaying the results of the buffering function. Image courtesy of GeoEye.

4. RBMapper interface displaying the results of the detect vegetation and detect forest functions. Image courtesy of GeoEye.

5. RBMapper interface displaying water detection of nonwater features. Image courtesy of GeoEye......11

6. Example of mixed water identification. Image courtesy of GeoEye. 


\title{
Development and Evaluation of a Riparian Buffer Mapping Tool
}

\author{
By Lesley E. Milheim and Peter R. Claggett
}

\section{Introduction}

Land use and land cover within riparian areas greatly affect the conditions of adjacent water features. In particular, riparian forests provide many environmental benefits, including nutrient uptake, bank stabilization, steam shading, sediment trapping, aquatic and terrestrial habitat, and stream organic matter. In contrast, residential and commercial development and associated transportation infrastructure increase pollutant and nutrient loading and change the hydrologic characteristics of the landscape, thereby affecting both water quality and habitat. Restoring riparian areas is a popular and cost effective restoration technique to improve and protect water quality. Recognizing this, the Chesapeake Executive Council committed to restoring 10,000 miles of riparian forest buffers throughout the Chesapeake Bay watershed by the year 2010 (Chesapeake Executive Council, 2003). In 2006, the Chesapeake Executive Council further committed to "using the best available...tools to identify areas where retention and expansion of forests is most needed to protect water quality" (Chesapeake Executive Council, 2006).

The Chesapeake Bay watershed encompasses 64,000 square miles, including portions of six States and Washington, D.C. Therefore, the interpretation of remotely sensed imagery provides the only effective technique for comprehensively evaluating riparian forest protection and restoration opportunities throughout the watershed. Although 30-meter-resolution land use and land cover data have proved useful on a regional scale, they have not been equally successful at providing the detail required for local-scale assessment of riparian area characteristics. Use of high-resolution imagery (HRI) provides sufficient detail for local-scale assessments, although at greater cost owing to the cost of the imagery and the skill and time required to process the data.

To facilitate the use of HRI for monitoring the extent of riparian forest buffers, the U.S. Forest Service (USFS) and the U.S. Geological Survey (USGS) Eastern Geographic Science Center funded the development of a prototype semiautomated image classification tool, RBMapper, that is designed for use by technicians with limited image processing training.

The remainder of this document provides an overview of the RBMapper tool, instructions on how to obtain the RBMapper tool and tutorial datasets, and a summary evaluation of the tool. Appendix 1 provides additional information from the reviewers.

\section{RBMapper Overview}

RBMapper was developed in 2006 by GDA Corporation in conjunction with the USGS and the USFS. RBMapper is a prototype image processing software tool that classifies land cover within riparian areas from HRI and ancillary hydrography and elevation datasets. While other software packages are available to perform image processing, they are complex and require highly 
skilled users to effectively process the HRI. RBMapper was developed to semiautomate the water delineation, buffering, and classification processes for users with limited training. It provides the following functions:

- Atmospheric adjustment and pan-sharpening of the image,

- Specialized algorithms for water detection,

- Edit functions for user correction of the detected water features,

- Buffer delineation based on topographic or Euclidean distance,

- Discrimination of general land cover classes (water, bare ground, herbaceous vegetation, forest) within the buffers, and

- Calculation of associated land cover statistics (for example, total areas of forest, ground vegetation, and bare ground).

An initial version of RBMapper was delivered to the USFS and the USGS in November 2006. The first round of tests identified numerous issues and problems, many of which were addressed. A stable version of RBMapper that was suitable for evaluation by a larger audience was released on July 6, 2007. This prototype version is the one referred to in this report.

\section{RBMapper Availability}

RBMapper Version 1.2.8 can be downloaded from the GDA Corporation Web site at http://gdacorp.com/projects/RBMapper.html. Instructions for downloading, installing, and registering the software and for obtaining tutorial datasets are also available from the Web site. The Web site allows access to digital copies of the user's manual, tutorial, and release notes. To use the RBMapper software, one is required to register the software and obtain a software key code from GDA Corporation. To use the software tutorial, one must download a copyrighted IKONOS image, courtesy of GeoEye, and ancillary datasets.

The tutorial datasets include an IKONOS image in GeoTIFF format of a location in eastern Kent County, Maryland, and ancillary data for the entire Chesapeake Bay watershed. The ancillary datasets include a 30-meter digital elevation model, a linear shapefile for streams, and a polygon shapefile for water bodies. The hydrography shapefiles were generated from 1:24,000 scale National Hydrological Dataset (NHD) and Digital Line Graph (DLG) files. The tutorial datasets are projected to UTM Zone 18, WGS 1984 datum.

\section{Evaluation of the RBMapper Software}

Following release of the prototype RBMapper in July 2007, the tool was reviewed by representatives of the Federal Government, State governments, and nongovernmental organizations to evaluate its potential for use by these organizations. The focus of the evaluation was on RBMapper's functionality, accuracy, and usability, especially for users with limited knowledge of and skill in processing remote-sensing data. Each of the reviewers completed the tutorial and then experimented with the tool's capabilities as those functions might be used in his or her official duties. Details on the evaluation and the reviewers' comments are provided in Appendix 1. 


\section{Strengths}

RBMapper provides fast reproducible estimates of forest canopy cover within user defined buffers of water features. RBMapper enables the correction of water features, which is a necessity when using 1:24,000 scale hydrography datasets with HRI. RBMapper is easily learned, has an intuitive user interface, and allows persons with limited remote-sensing experience to process images covering large areas relatively quickly. The accuracy and quality of the output are highly dependent on the amount of water feature correction required, how much of that correction is performed, and the skill with which RBMapper functions are used. The reviewers agreed that RBMapper provides satisfactory results given a minimum level of training.

\section{Weaknesses}

RBMapper's primary weaknesses are instability, uneven automated detection of water features, variability of results based on user knowledge and experience, and dependence on IKONOS imagery. Such issues as software instability and data dependence are common in the prototype phase of software development, and although most of the instability issues were resolved prior to release of the prototype release in July 2007, some issues remained unresolved.

There are obvious tradeoffs between software usability and functionality. In developing the RBMapper, the challenge was to maximize functionality while not compromising usability by persons with limited training. Given the variability in image quality, even for images from the same platform, it was necessary to add functionality at the cost of requiring increased user knowledge and experience. RBMapper was designed to work only with IKONOS imagery to minimize the problem of image quality on the software development process for this proof-of-concept tool. Although the exclusive use of IKONOS imagery provides the desired level of spectral and spatial resolution, the cost of the imagery may preclude its use by local governments and watershed organizations, especially because aerial imagery is often available to them at little or no cost.

\section{Conclusions}

The RBMapper is new software that provides a semiautomated approach to mapping riparian forest buffers from HRI and requires minimal technical experience on the part of users. While it is only prototype software, it can be used in its current state by Federal, State, and local Chesapeake Bay Program Partners to map riparian buffers. The USGS and the USFS are exploring further refinements to the tool to increase its functionality, accuracy, and usability by local governments and nongovernmental organizations that lack remote-sensing expertise and software. Planned refinements to the RBMapper include (1) use with color-infrared aerial photography; (2) wall-to-wall mapping of tree canopy, herbaceous vegetation, and bare ground; and (3) clarification of the user interface.

\section{Acknowledgments}

We gratefully acknowledge the participation of our independent reviewers, who are as follows: Harry Berquist, Virginia Institute of Marine Science; Seth Coffman, Potomac Conservancy; Fred Irani, Maryland Department of Natural Resources; Leroy Ledon, University of Puerto Rico; Jarlath O’Neil-Dunne, University of Vermont; Stephanie Orndorff, The Nature Conservancy; James Pugh, Virginia Department of Forestry; Chris Robinson, NOAA C-CAP; Vanessa Torres, University of Puerto Rico. 


\section{References Cited}

Chesapeake Executive Council, 2003. Expanded riparian forest buffer goals: Chesapeake Executive Council Directive No. 03-01, 2 p.

Chesapeake Executive Council, 2006, Protecting the forests of the Chesapeake watershed: Chesapeake Executive Council Directive No. 06-1, 2 p. 


\section{Appendix 1-RBMapper Evaluation and Reviewer Comments}

The original agreement between GDA Corporation and the USGS Eastern Geographic Science Center described the development of prototype software to test the automation of mapping riparian buffers. As a prototype, it was understood that the software would likely not be suitable for use in a production environment without further refinements and error checking. The original goal was to simplify and streamline riparian buffer mapping by developing new algorithms and tools, which, combined with IDRISI (Clark Labs, Clark University) raster geographic information system (GIS) software to provide visualization and editing capabilities, could do the following automatically: (1) perform at-sensor correction of the imagery; (2) subset and reproject any ancillary data to the extent, resolution, and coordinate system of the image; (3) pan-sharpen the imagery; (4) use spectral, textural, and contextual information in mapping the riparian buffers; and (5) map riparian buffers using terrain to estimate the distance from the water. During development of the prototype, however, GDA recommended developing a custom visualization interface and abandoning the proprietary platform (IDRISI) for visualization. This recommendation was accepted by the USGS and the USFS and resulted in a more cohesive product. This change raised reviewer expectations that the RBMapper would function with an intuitive user interface and minimum errors rather than as a prototype of what could be achieved through additional refinements.

RBMapper was evaluated by nine individuals with a wide range of skills, knowledge, and experience in mapping riparian buffers. Two reviewers were summer interns with classroom experience using GIS but no remote sensing experience. They learned about RBMapper and ERDAS Imagine (an image processing software package produced by Leica Geosystems LLC) using tutorials and then compared their ability to process the same images using both tools.

The remaining seven reviewers are highly trained and skilled professionals, each with 5 to 25 years of experience with GIS and remote sensing. These individuals represent Federal and State agencies, nongovernmental organizations, and one university. These reviewers all used the tutorial and the tutorial data to evaluate RBMapper. One reviewer also performed a comparison between RBMapper with ERDAS Imagine techniques used by his agency. Many of the other reviewers discussed their expectations of RBMapper given their experience with other image processing tools.

\section{Results}

While individual opinions differed on various implementation details, particularly regarding the user interface, all the reviewers agreed that RBMapper provides reasonable results without great difficulty and that it is easily used by those without significant training in remote sensing and image processing.

RBMapper was developed for use by those with limited skill and experience in image processing; nonetheless, training is required to effectively use RBMapper. The summer interns struggled with RBMapper and seemed to have more trouble with RBMapper shutting down than the other reviewers. However, they found it easier to use than the more sophisticated image processing tools. Those who professionally research land cover using image processing techniques acknowledged that the tool has benefit, especially for those with limited remote sensing and GIS experience; given their level of expertise and professional goals, however, these researchers felt that the more sophisticated image processing software packages were more suitable for their purposes. 


\section{Usability}

Generally, RBMapper works as described in the user's manual and in the tutorial. Many reviewers commented on the intuitive interface. Figure 1 shows the interface with the windows for setting preferences open.

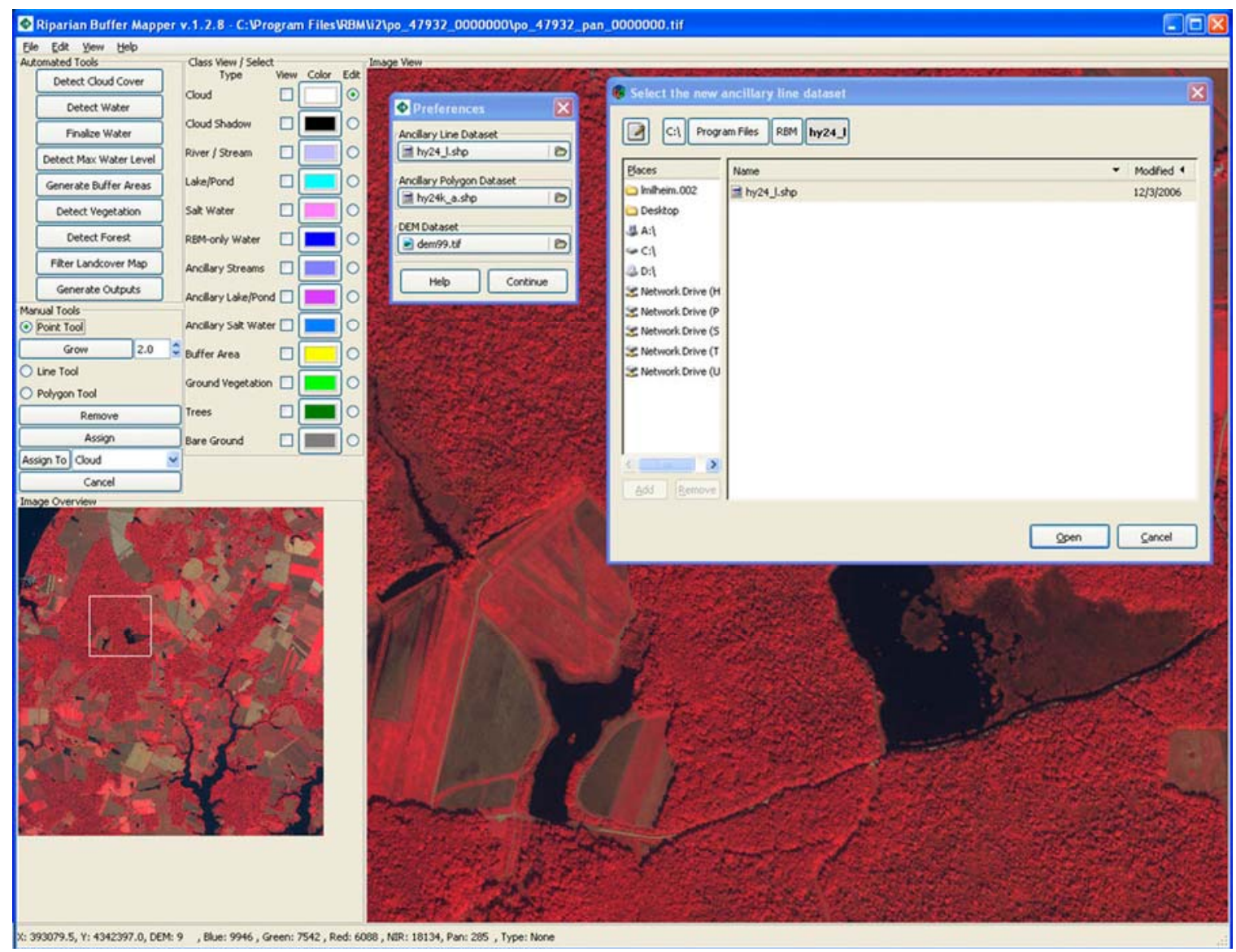

Figure 1. RBMapper interface displaying the tutorial image with the windows for setting preferences open. Tutorial image courtesy of GeoEye.

RBMapper assumes the use of default pathnames for the tutorial data, although the user can specify new pathnames and data. RBMapper controls are accessed through the use of buttons located in the upper left portion of the window. The controls are invoked using a series of function buttons for processing the image and for editing the ancillary data. The functions produce and operate on the color-coded data layers located immediately to the right of the control buttons. The function buttons allow the user to detect cloud cover (not implemented at this time), detect water, finalize the water mask, detect the maximum water level, generate the buffer area, detect vegetation, detect forest, filter the land cover classes, and generate land cover output statistics. These functions are intended to be used in sequence, although the user can go back and re-execute a step to refine the process, but this will cause the previous results to be lost. 
RBMapper automates the image processing functions to detect water, to buffer that water, and to determine land cover within those buffers (figs. 2-4). Most of the experienced reviewers wanted more information on the parameters and the algorithms used in water detection and land cover classification so that they could better understand and control the process.

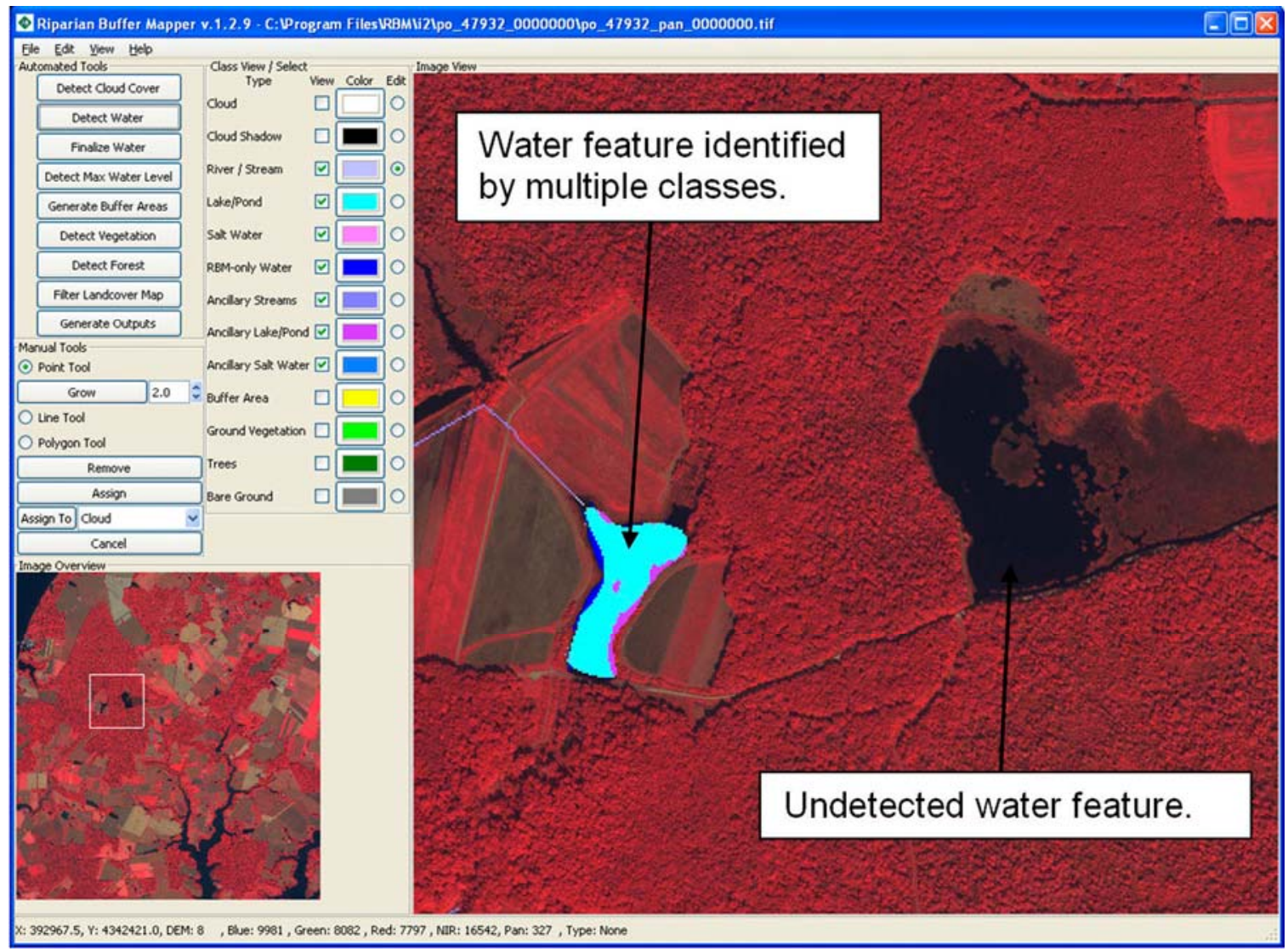

Figure 2. RBMapper interface displaying the results of the water detection function using default settings. Image courtesy of GeoEye. 


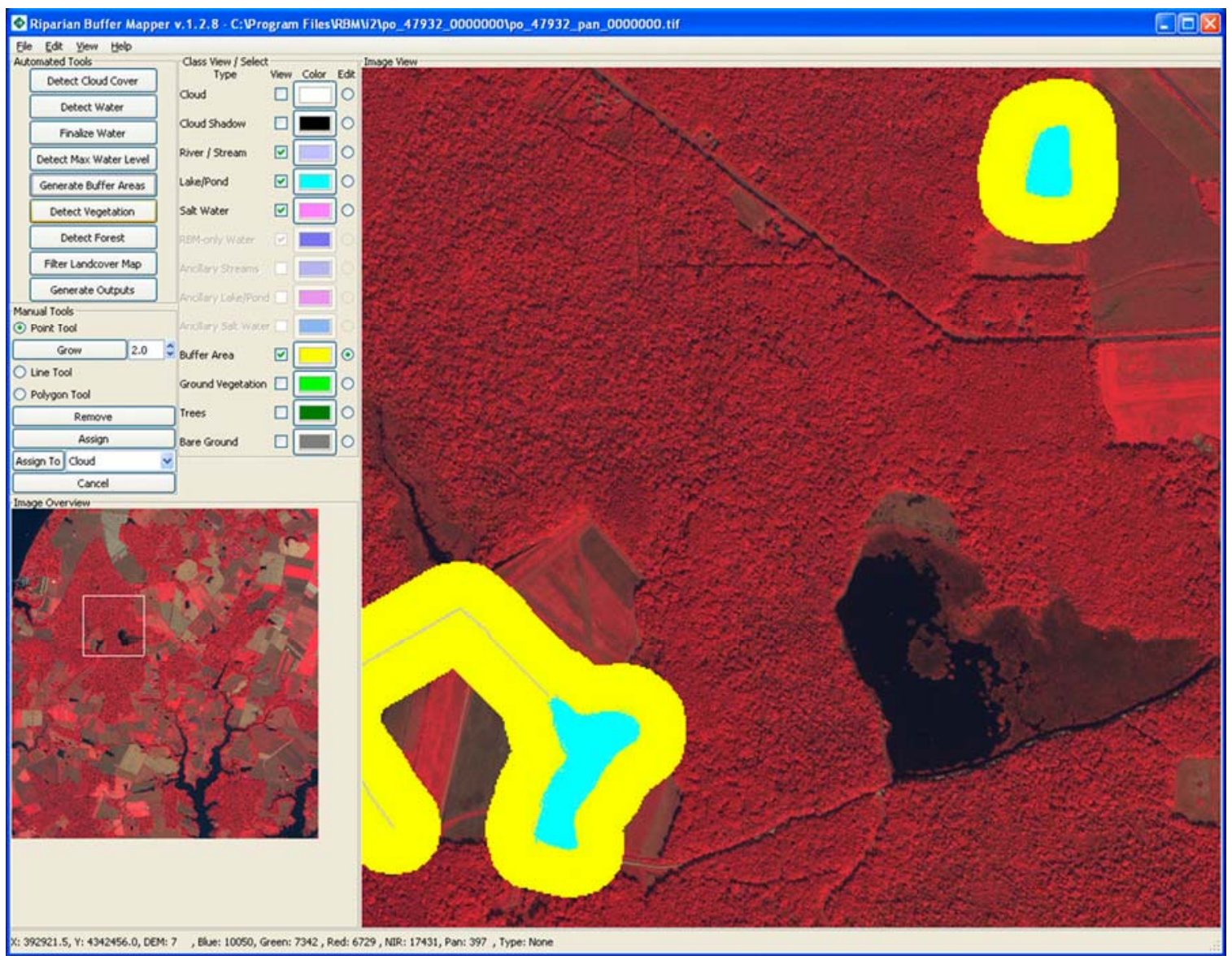

Figure 3. RBMapper interface displaying the results of the buffering function. Image courtesy of GeoEye. 


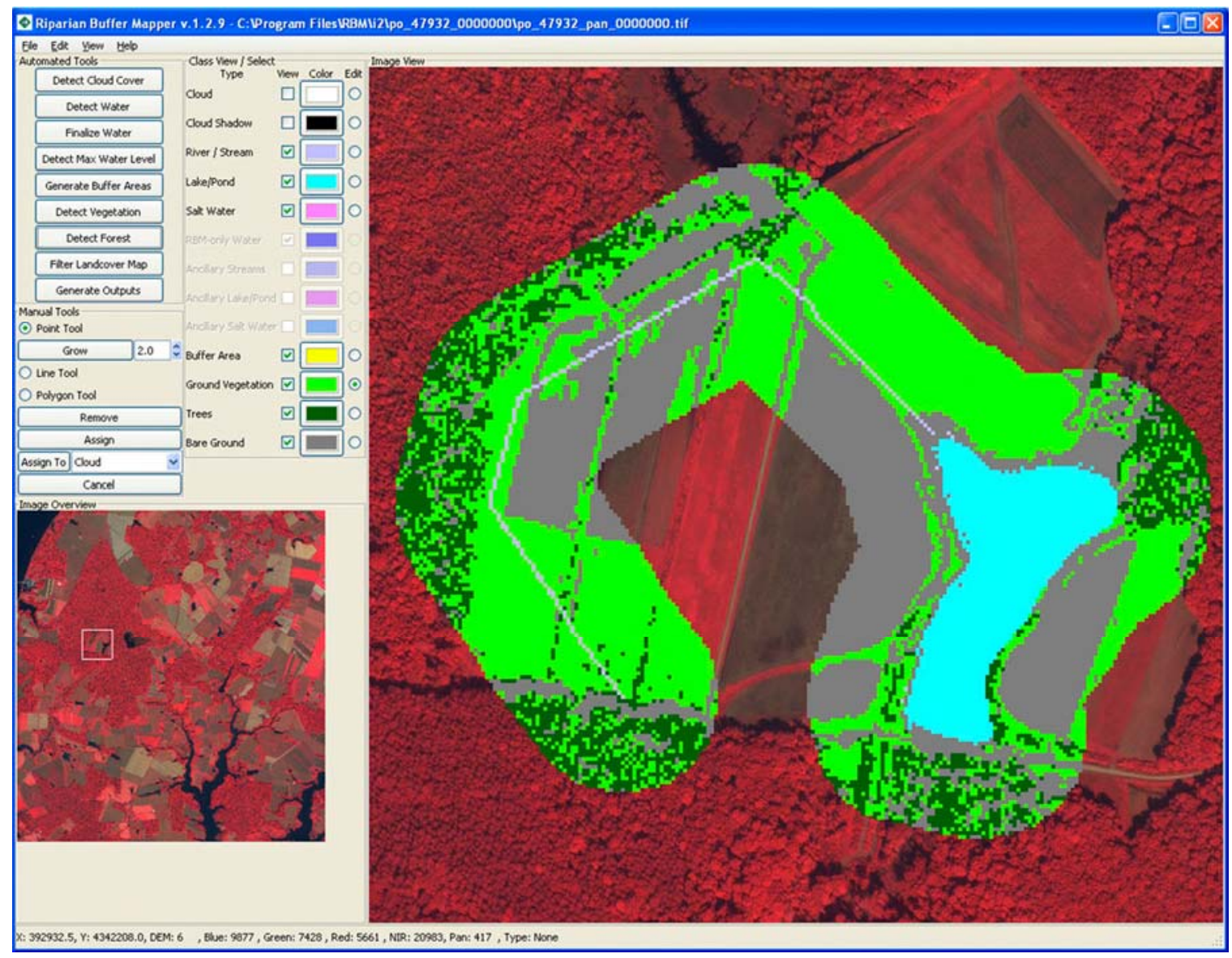

Figure 4. RBMapper interface displaying the results of the detect vegetation and detect forest functions. Image courtesy of GeoEye.

\section{Operational Issues}

The most common concerns raised about the RBMapper relate to its stability, the inability to save user inputs for future processing, restrictive imagery requirements, use of 1:24,000 scale hydrography data, the question of performance over a variety of physiographic provinces, and the level of expertise and effort required to develop a high-resolution water feature dataset. These issues are explained in greater detail below.

Software Instability. RBMapper occasionally and unexpectedly shuts down during processing. There is no consistent pattern that leads to an unexpected shutdown. Instability causes lost time in that data are not retained and must be regenerated after RBMapper shuts down unexpectedly. The user can save the project frequently, but this does not alleviate the time lost in restarting the tool. The stability issue is complicated by the fact that it may be related to the training of and shared expectations between the developers and the professional-level users. The less knowledgeable users who do not share this background may unknowingly make mistakes that cause errors within RBMapper.

Inability to Save Preferences. RBMapper assumes a default location for the image and ancillary data files. The user may specify an alternative pathname and filename by selecting File $>$ 
Preferences and navigating to the appropriate pathname for each of the three ancillary datasets. However, these alternative locations are not saved and restored when the RBMapper project is saved and re-opened, so the user must respecify the preferences for subsequent work. Similarly, the RBMapper functions assume default parameters that the user may change, but these changes are not saved for repeated use of the function.

Restrictive Imagery Requirements. The exclusive use of IKONOS imagery can also be considered a weakness. Although this imagery does provide the desired level of spectral and spatial resolution, the imagery expense is difficult for the intended user community to justify because many local government users have existing aerial imagery or other spectral data available to them at less or no cost.

Scale of Data Can Be Problematic. High-resolution hydrography data of less than 1:5,000 scale are needed to align well with water features identifiable using such high-resolution imagery as IKONOS. The hybrid 1:24,000 scale National Hydrography Dataset - Digital Line Graphs ancillary dataset $^{1}$ used with the RBMapper requires significant editing to align it with water features identified from IKONOS imagery. The amount of editing is particularly significant in urbanizing areas where water features may be greatly altered from their original mapped state. The hybrid hydrography dataset is also limiting because it includes a water identification field (for example, freshwater, or saltwater) that is not available in the standard NHD dataset. The use of nonstandard fields to describe water features requires the user either to use the tutorial hydrography dataset with the user-supplied imagery or to modify a local hydrography dataset to include the necessary data fields for use with RBMapper.

The Chesapeake Bay watershed is very large, encompassing parts of four major physiographic regions: coastal plain, piedmont, ridge and valley, and Appalachian plateau. Land cover and water feature patterns and densities vary across these regions, which may impact RBMapper performance. The RBMapper has yet to be systematically tested in different ecoregions. Each ecoregion may need different default parameter settings to optimize land cover classification.

Higher-Than-Expected Level of Expertise Required for Some Functions. The higherthan-expected level of expertise and effort required to operate RBMapper may be seen in (1) the extensive corrections to the water detection results that may be needed to create the water mask, (2) the access to and knowledge of a GIS to create additional fields required in the hydrography dataset, and (3) the knowledge of parameter effects on the RBMapper functions. Additional documentation on parameters and processing algorithms would alleviate the latter comment.

The uneven detection of water features and inaccuracies within the ancillary data require the user to correct the water mask before generating the buffers and classifying the land cover within those buffers. RBMapper creates a water mask using a water detection algorithm and the stream and water body datasets. The mask then serves as input to the buffering function. However, water is not always detected (fig. 2) or is detected where there is no water (fig. 5). Alternatively, the stream and water bodies are misplaced because of ancillary data accuracy issues or land cover change between the source date of the ancillary data and the source date of the image. These problems can require significant correction to the water mask. Such correction may be a multistep process. For example, RBMapper may classify a lake as five water classes: ancillary lake/pond class, lake/pond class, ancillary river/stream (feature boundary) class, river/stream class, and water class. The simplest way to correct this feature is to delete pixels belonging to each of the classes in separate

\footnotetext{
${ }^{1}$ At the time the RBMapper was developed, the 1:24,000 scale NHD was not yet complete for the entire Chesapeake Bay watershed.
} 
steps, then to use the "grow" function to identify the actual feature from the imagery reflectance values. Similar problems occur where streams cross roads.

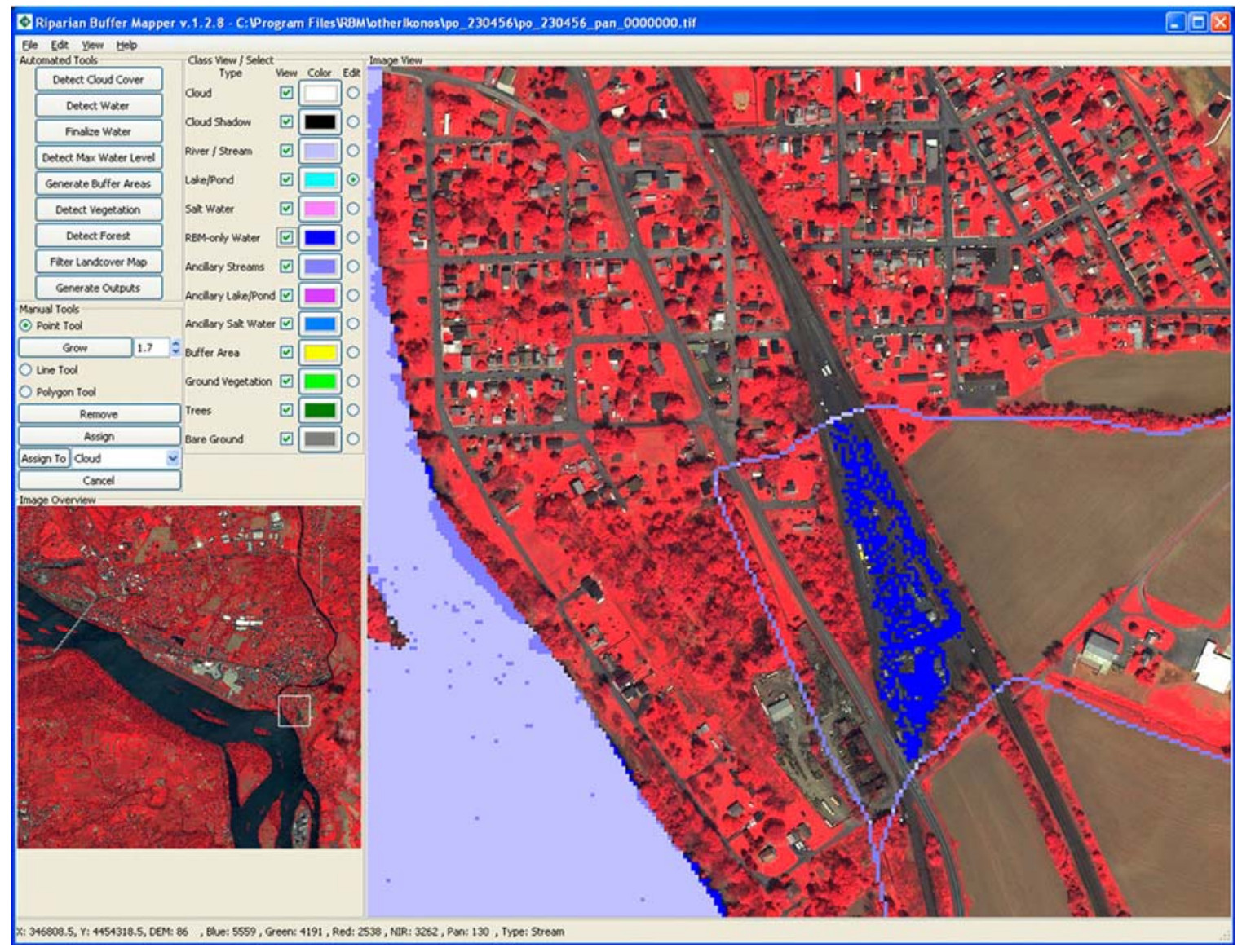

Figure 5. RBMapper interface displaying water detection of nonwater features. Image courtesy of GeoEye.

The correction of the water mask may be substantial, depending on the terrain and the amount of development since the collection of the ancillary data. Seasoned professionals may not notice the effort required to correct the water mask, but it may cause difficulty for nonprofessional users. Making similar corrections to the water mask during subsequent analyses of the same area for different time periods may affect temporal trend statistics because spectral interpretation may delineate larger or smaller water areas or configurations depending on in situ conditions for those time periods.

The use of nonstandard hydrologic data requires a higher level of training and access to software tools to modify the data than is implied by the manuals. Other aspects of the software, such as parameters for water detection, the "grow" function, and land cover class differentiation require a greater understanding of remote sensing than is intended for this application. Software characteristics such as these increase the level of training and experience needed to achieve highquality results using RBMapper. 


\section{Accuracy}

The accuracy and quality of the RBMapper output are highly dependent on the amount of correction required to complete the water mask, how much of that correction is performed, and the skill with which the RBMapper functions are used. However, the reviewers agreed that, given the level of training required, RBMapper generates adequate results. This section summarizes the comments on the accuracy of processing the tutorial image, differences across physiographic provinces, and the effects of hydrography datasets of different resolutions.

Several reviewers commented on the presumed accuracy of the output because of the similarity in techniques between RBMapper and in-house image-processing procedures, but only one reviewer actually performed a direct comparison. This reviewer compared the results of riparian buffer mapping using RBMapper with an in-house technique using ERDAS 8.7 (Leica Geosystems LLC) and eCognition 5.0 (Definiens). The technique using eCognition 5.0 takes about 1 hour, but requires substantial training. The technique using RBMapper also took about 1 hour, but the RBMapper produced more forest speckling (even though filtering was used), and the output shows more confusion with agricultural areas.

A visual inspection of land cover classified using default settings compared with the source image reveals that what is classified as vegetation is typically vegetation; the forest class, however, may represent either forest or vegetation and the bare ground class may represent bare ground, impervious surfaces, or vegetation. In an urbanized setting, this confusion in classification and hence in the land cover statistics could be misleading, even though the goal is to identify tree crowns near streams and not bare ground or impervious surfaces. These classifications may be altered by manipulating the classification parameters, but this manipulation requires knowledge of those parameters and their effects.

One reviewer processed images from three physiographic regions: one image each from Kent and Talbot Counties, Maryland, in the coastal plain; one image from Dauphin County, Pennsylvania, in the Appalachian uplands; and one image from Frederick County, Maryland, in the piedmont. In general, the results were similar. However, the image from Pennsylvania required more significant correction of the hydrography data than the other images. This difference may be because of the greater amount of urbanization shown in that image and the presence of more braided and meandering stream beds. An interesting result of using a hydrography dataset for other than its original purpose was the classification of islands in the Susquehanna River as lakes. The islands appear as polygons in the river, but there is no provision for identifying a land structure in this dataset and RBMapper interprets unspecified polygons as fresh water bodies.

An early concern in evaluating RBMapper was the resolution of the tutorial's elevation and hydrography data. The hydrologic data are mapped at 1:24,000 scale and the elevation is 30-meter resolution whereas the IKONOS imagery contains 4-meter resolution multispectral data sharpened to 1-meter resolution using the panchromatic band. Several reviewers commented that the use of higher resolution ancillary data would improve the results of RBMapper.

However, when RBMapper was evaluated using synthetic stream data generated using elevations of varying resolution. It was found that the use of higher resolution stream data did not significantly change the land cover statistics calculated by RBMapper, although, the number of gaps did increase with the higher resolution stream data. The expected differences may not have manifested because of the homogeneity of the test landscape or because the same water body dataset was used for all tests. Therefore, the results of this experiment are inconclusive. 


\section{Additional Comments}

A few reviewers provided additional comments or summary statements, such as the following:

1. There is greater data analysis capability with other software packages, but they also require more knowledge of remote sensing and greater effort. RBMapper provides a straightforward approach to extracting forest vegetation within user-specified buffers. I prefer eCognition (Definiens), but see applicability of RBMapper for resource managers or planners with little or no remote-sensing knowledge.

2. An intern liked RBMapper's more user-friendly program for one of limited experience.

3. RBMapper must be used with process standards, such as whether or not to include bridges when correcting the water mask and how small a stream or pond to include. These items have the potential to significantly affect the buffer statistics and require a consistency in the buffer definition and classification.

4. The homogeneous conditions in the tutorial imagery represent a best case scenario for mapping land cover. Pixel based classifiers work less well under more heterogeneous conditions.

5. The tutorial datasets were conveniently all of the same projection. While RBMapper will project all datasets to the image's projection on-the-fly, it is a time-consuming process.

6. The user must also be aware that intermediate files are kept in the output folder within the IKONOS imagery folder. If the user wishes to restart the riparian buffer mapping process from the beginning, he or she should delete the output folder and its contents before restarting. Otherwise, those intermediate files are re-used.

7. The distinction between the data layers is not clear. For instance, what distinguishes the lake/pond layer from the ancillary lake/pond layer? The speckling of these layers with the RBMapper-detected water layer makes the water mask correction more difficult than it needs to be (fig. 6). This point was emphasized by one reviewer who created an empty water body dataset and removed the boundaries of large water features (for example, a broad river) that extend outside the image footprint from the stream dataset, leaving the smaller streams delineated by a single line, and then used these alternative datasets to process the image. This technique greatly reduced the amount of correction because RBMapper (1) grew the water features with good boundaries that needed no correction, although not all water bodies were detected, as mentioned earlier; and (2) did not identify water bodies with as many mixed classes. 


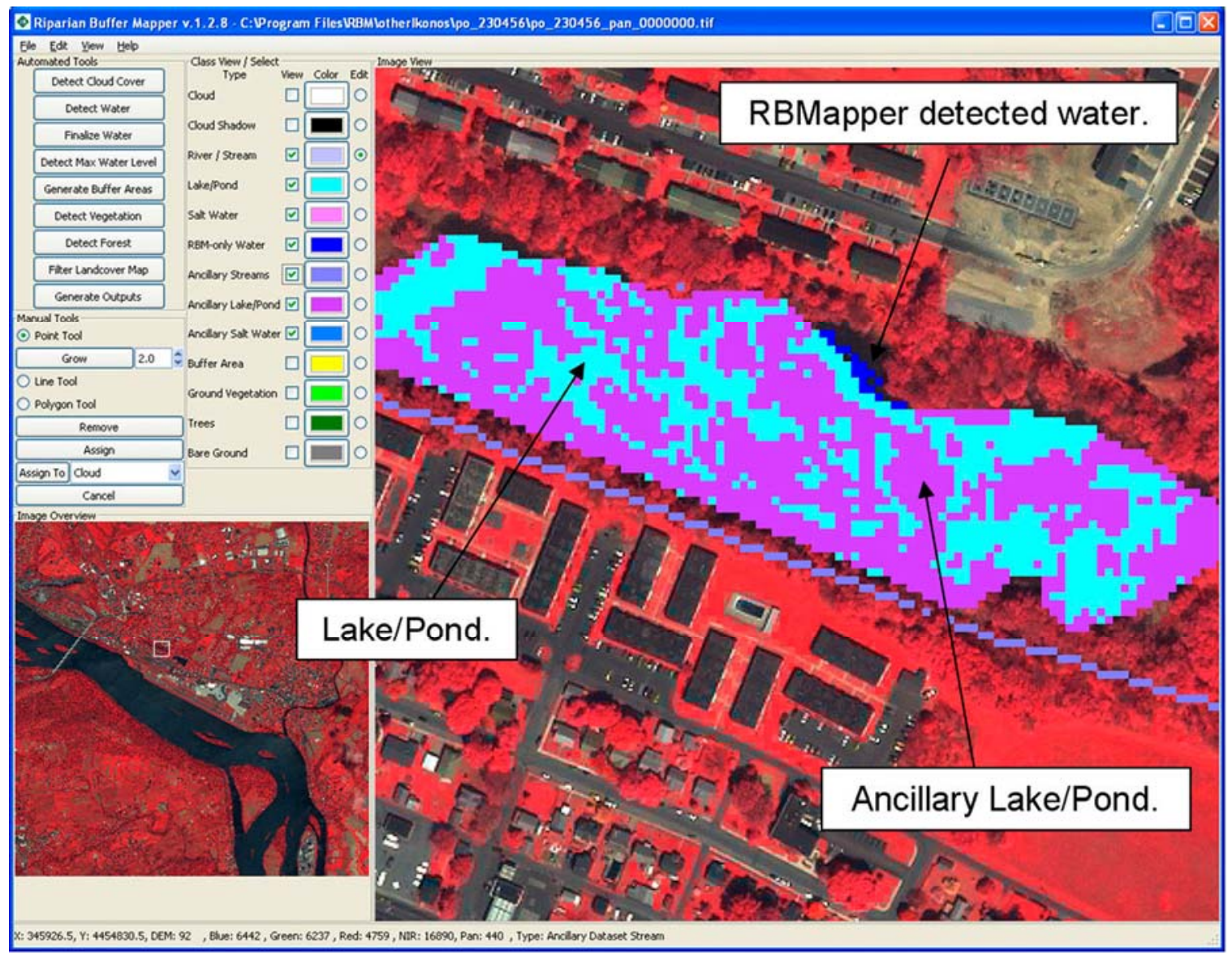

Figure 6. Example of mixed water identification. Image courtesy of GeoEye.

\section{Conclusions}

The reviewers may have had unrealistic expectations of the software because six of them were highly trained technicians who were accustomed to using very flexible image processing software (for example, ERDAS Imagine, ENVI, Definiens) with minimal errors and detailed help and tutorial files. Two of the reviewers with minimal training were students and appeared to struggle most with the tool, in part because they had little understanding of the purpose of mapping riparian buffers. One of the remote sensing technicians found the tool potentially useful, but expressed the need to use it with aerial photography that is freely available. The use of IKONOS imagery limited the evaluation of almost all reviewers to the tutorial dataset.

Remote-sensing technicians tended to prefer their current image classification software and were disinclined to use less flexible tools like the RBMapper. Therefore, those with limited experience and knowledge in remote sensing may be more appropriate users of RBMapper. However, the need to build nonstandard hydrologic datasets, to correct the water mask, and to "tinker" with the details is nontrivial and may deter use of the tool by less trained users. Input from such potential users (for example, resource managers or planners) on RBMapper's ability to provide a needed service is vital. 
Assuming continued RBMapper development, the following issues identified in this evaluation and in the initial testing should be addressed: (1) The interface and its multiple classification layers, including the use of the nonstandard hydrologic data, need to be clarified and documented, and perhaps redesigned; (2) The processing of alternative high-resolution imagery and datasets needs to be evaluated; (3) The processing standards for use with RBMapper, such as a minimum mapping unit, need to be identified; (4) An accuracy assessment needs to be performed; and (5) RBMapper should be tested over all major physiographic provinces in the Chesapeake Bay watershed to identify any necessary changes in default parameter settings. 